EPJ Web of Conferences 108, 02039 (2016)

DOI: $10.1051 /$ epjconf/201610802039

(C) Owned by the authors, published by EDP Sciences, 2016

\title{
Quasiclassical Approximation in the Non-Relativistic and Relativistic Problems of Tunneling lonization of a Hydrogen-Like Atom in a Uniform Electric Field
}

\author{
O. K. Reity ${ }^{1, a}$, V. K. Reity ${ }^{1, b}$, and V. Yu. Lazur ${ }^{1, c}$ \\ ${ }^{1}$ Department of Theoretical Physics, Uzhhorod National University, 54 Voloshyna Street, Uzhhorod 88000, \\ Ukraine
}

\begin{abstract}
A recurrent scheme for finding the quasiclassical solution of the onedimensional equation obtained after the separation of variables in the Schrödinger equation in parabolic coordinates is derived. The method of quasiclassical localized states is developed for the Dirac equation with an arbitrary axially symmetric potential of barrier type which does not allow complete separation of the variables. By means of the proposed quasiclassical methods the non-relativistic and relativistic wavefunctions for hydrogenlike (H-like) atoms in an external uniform electrostatic field of intensity $F$ are constructed in the classically forbidden and allowed regions. The general analytical expressions of the leading term of the asymptotic behaviour (at small $F$ ) of the ionization rate of an $\mathrm{H}$-like atom in the uniform electrostatic field are obtained for the non-relativistic and relativistic cases.
\end{abstract}

\section{Introduction}

The problem of the hydrogen atom in an electric field plays a fundamental role in quantum mechanics and atomic physics and has many applications (see, e.g., [1-3] and the references therein). Since the twenties (see, e.g., the review [4]), the properties of the energy spectrum of the hydrogen atom and other atoms in external fields were rather intensively studied in the framework of the Schrödinger equation.

At the same time the logic of development of the studies of highly ionized atomic systems demands the formulations of new problems, similar to those already solved only for neutral (or weakly ionized) atomic systems. The relativistic character the of electron motion in fields created by multiply charged ions (the characteristic velocity of the electron in $\mathrm{H}$-like ions with nuclear charge $Z$ is $\sim \alpha Z c ; \alpha$ is the fine structure constant, $c$ is the velocity of light) distinguishes them drastically from the neutral atoms. Thus, the consistent theory of the tunneling ionization of such systems should be essentially relativistic since the relativistic effects are not small in this case, and moreover they determine the order of magnitude of the spectral characteristics.

In order to construct such a theory one should employ the solution of the relativistic problem of the electronic motion in the field created by the nucleus and a constant uniform electric field. Since the

\footnotetext{
ae-mail: oleksandr.reity@uzhnu.edu.ua

be-mail: 13reity@gmail.com

ce-mail: volodymyr.lazur@uzhnu.edu.ua
} 
Dirac equation with such superpositional potential does not permit complete separation of variables in any orthogonal system of coordinates, the given problem has not exact analytical solution, and numerical methods are needed which demand significant computational efforts.

Relativistic calculations of the linear Stark effect were carried out by means of perturbation theory $[5,6]$, and the quadratic Stark effect was treated by means of the RCGF (Relativistic Coulomb Green Function) method in the form of the expansion in powers of $Z \alpha$ [7]. However, the publications in this field are basically devoted to the calculation of the position of a quasistationary level, and there are only rare cases of calculation of level widths $\Gamma=\hbar w(w$ is the tunneling ionization rate) in the relativistic case. In our previous paper [8] a hybrid version of a spherically symmetrical model of the Stark effect with account the Lorentz structure of the interaction potential has been studied in the quasiclassical approximation. The ionization rate of the $s$-level, the binding energy of which can be of the order of the rest energy in electric and magnetic fields has been calculated by means of a generalization of the imaginary time method (ITM) [9] and the so-called ADK-theory [10]. However, in the general case, the widths of the quasistationary states have not been found until now.

Due to such situation in the theory and the intensive experimental researches during the last years, asymptotic methods for the calculation of ionization rates, which are based on clear physical ideas describing the under-the-barrier electronic transition, become especially important. From this point of view it is worthwhile to use the quasiclassical approximation which enables one to find the approximative analytical solutions of the relativistic problem and to express the required ionization probability in terms of the quantum penetrability of the potential barrier which separates the domains of discrete and continuous spectra. As it is known, this method has a rather high accuracy even for small quantum numbers.

In the present paper we apply the quasiclassial approximation to both the non-relativistic and relativistic problems of tunneling ionization of $\mathrm{H}$-like ions in a constant uniform electric field. The first problem is much simpler than the second one due to the separability of the Schrödinger equation in parabolic coordinates. In this problem we can use the expansion in powers of the Planck constant $\hbar$. For the relativistic problem we apply the method of quasiclassical localized states for the Dirac equation with axially symmetrical potential the basics of which were described in [11].

\section{Quasiclassical solutions of the non-relativistic problem of the atom in a constant uniform electric field}

The potential of an $\mathrm{H}$-like atom with charge $Z$ in a constant uniform electric field (the intensity vector $\vec{F}$ of which is opposite to the axis $z$ ) can be represented in the form $\left(\hbar=e=m_{e}=1\right)$

$$
V=-Z / r-F z
$$

As it is known [1], the Schrödinger equation with the potential (1) permits complete separation of variables in parabolic coordinates $\xi=r+z, \eta=r-z, \phi=\operatorname{arctg}(y / x)$. For this purpose we seek the solution in the form

$$
\Psi=(\xi \eta)^{-1 / 2} \varphi(\xi) \chi(\eta) e^{ \pm i m \phi},
$$

where $m=0,1,2, \ldots$ is the absolute value of the magnetic quantum number. Substituting (2) into the Schrödinger equation, we obtain the following equations for the unknown functions $\varphi(\xi)$ and $\chi(\eta)$ :

$$
\begin{aligned}
& \frac{d^{2} \varphi}{d \xi^{2}}+\left(\frac{E}{2}+\frac{\beta_{1}}{\xi}+\frac{1-m^{2}}{4 \xi^{2}}+\frac{F}{4} \xi\right) \varphi=0, \\
& \frac{d^{2} \chi}{d \eta^{2}}+\left(\frac{E}{2}+\frac{\beta_{2}}{\eta}+\frac{1-m^{2}}{4 \eta^{2}}-\frac{F}{4} \eta\right) \chi=0, \quad \beta_{1}+\beta_{2}=Z .
\end{aligned}
$$


For the energy $E$ of a quasistationary level we shall use the known expansion [12]

$$
E=-\frac{Z^{2}}{2 n^{2}}-\frac{3 n\left(n_{1}-n_{2}\right) F}{2 Z}+O\left(F^{2}\right)
$$

where $n=n_{1}+n_{2}+m+1$ is the principal quantum number.

Within the perturbation theory Damburg and Kolosov [13] have found the asymptotic (at small $F$ ) solutions of the equations (3), (4). Substituting them into (2), normalizing the wavefunction $\Psi$ to unity and taking into account only the leading terms in $F \ll 1$ and $\xi^{-1} \ll 1$ we obtain

$$
\begin{gathered}
\varphi_{0}=C_{0}(\gamma \xi)^{n_{1}+m / 2} e^{-\gamma \xi / 2}, \quad \chi_{0}=(\gamma \eta)^{m / 2} e^{-\gamma \eta / 2} L_{n_{2}}^{m}(\gamma \eta), \\
C_{0}=(-1)^{n_{1}} \sqrt{\frac{n_{2} ! \gamma^{3}}{\pi n n_{1} !\left(n_{1}+m\right) !\left(n_{2}+m\right) !}},
\end{gathered}
$$

$\gamma=\sqrt{-2 E}, L_{k}^{m}(x)$ is the Laguerre polynomial.

The solutions of the equation (3) at large $\xi$ are oscillating and the formula (6) is unapplicable here. The limitation of the applicability of the solution (6) is the standard requirement of smallness of the perturbation $F \xi / 4$ as compared to the "Coulomb field" $\beta_{1} / \xi$. This gives the condition $0 \leqslant \xi \ll \xi_{m}$ where $\xi_{m}=\sqrt{4 \beta_{1} / F}$ is the point in which contributions of the "Coulomb field" and external electric one in the "potential energy" $U_{1}(\xi)$ are equal to each other.

In [13-15], the construction of solutions beyond the range $0 \leqslant \xi \ll \xi_{m}$ uses the etalon equation method. This method is quite cumbersome in applications, especially when higher corrections are to be found. We use here the simpler WKB method to derive the asymptotic expression for the ionization rate $w$ under a region of applicability which is larger than that of the etalon equation method. Note that Smirnov and Chibisov [16] used the quasiclassical approximation but their result for $\gamma$ is incorrect because the multiplication factor $\exp \left[3\left(n_{2}-n_{1}\right)\right]$ is missing.

We seek the solution of (3) in the form,

$$
\varphi=e^{S / \hbar} \sum_{n=0}^{\infty} \hbar^{n} \varphi^{(n)} .
$$

Having substituted (7) into (3), temporarily restoring the Planck constant $\hbar$ and equating to zero the coefficients of each power of $\hbar$, we arrive at an hierarchically ordered set of equations for the unknown functions $S(\xi)$ and $\varphi^{(n)}(\xi)$, which are analytically solvable:

$$
\begin{aligned}
S(\xi) & =-\int_{\xi_{1}}^{\xi} q\left(\xi^{\prime}\right) d \xi^{\prime}, \quad \varphi^{(0)}=\frac{C^{(0)}}{\sqrt{q}}, \\
\varphi^{(n)} & =\frac{1}{\sqrt{q}}\left[\int \frac{1}{2 \sqrt{q}}\left(\varphi^{(n-1) \prime \prime}-\frac{m^{2}-1}{4 \xi^{2}} \varphi^{(n-1)}\right) d \xi+C^{(n)}\right],
\end{aligned}
$$

where $n=1,2, \ldots, C^{(n)}$ are arbitrary constants, $q=\sqrt{2(U-E / 4)}$, the function $U(\xi)=-\beta_{1} / 2 \xi-F \xi / 8$ plays a role of an effective potential energy.

According to the general conditions of applicability of the quasiclassical approximation [1], the potential barrier should be quite wide $\left(\xi_{1} \ll \xi_{2}\right.$, where $\xi_{1,2}$ are roots of equation $\left.q(\xi)=0\right)$. This gives the requirement $16 \beta_{1} F / \gamma^{4} \ll 1$, and the range $\xi_{1} \ll \xi \ll \xi_{m}$ exists $\left(\xi_{m}\right.$ is the maximum point of the potential barrier) where one can match the WKB solution (7) with the asymptotic behaviour (6):

$$
\varphi_{\xi_{1} \ll \xi \ll \xi_{m}}^{\simeq} \varphi_{0}
$$




\section{EPJ Web of Conferences}

Using (10) we obtain the normalized wavefunction in the under-the-barrier region. Using the Zwaan rule [1], this wavefunction can be continued in the classically allowed region $\xi>\xi_{2}$ where the divergent wave corresponds to the quasistationary state (hereinafter $\hbar=1$ again)

$$
\begin{gathered}
\varphi(\xi)=\frac{C^{(0)} \exp \left[-\int_{\xi_{1}}^{\xi_{2}} q(\xi) d \xi\right]}{\sqrt{p(\xi)}} \exp \left\{i \int_{\xi_{2}}^{\xi} p\left(\xi^{\prime}\right) d \xi^{\prime}+\frac{i \pi}{4}\right\}, \\
C^{(0)}=\frac{(-1)^{n_{1}} C_{0}}{n_{1} ! \sqrt{2}}\left(\frac{\beta_{1}}{\gamma}\right)^{n_{1}+(m+1) / 2} e^{-\beta_{1} / \gamma}, \quad p=i q=\frac{1}{2} \sqrt{F \xi-\gamma^{2}+\frac{4 \beta_{1}}{\xi}} .
\end{gathered}
$$

The probability of the system decay in the unit of time (or ionization rate) $w$ is determined as the total probability flux through a surface separating the H-like atom from outer part of the space [1]. Substituting (2), (6), (11), (12) into the expression of the ionization rate [1], we get

$$
w=\frac{Z^{2}}{n^{3} n_{1} !\left(n_{1}+m\right) !}\left(\frac{\beta_{1}}{\gamma e}\right)^{2 n_{1}+m+1} e^{-2 J}, \quad J=\int_{\xi_{1}}^{\xi_{2}} q(\xi) d \xi .
$$

The barrier integral $J$ can be calculated in terms of the complete elliptic integrals of 1-st and 2-nd kind, $K(k)$ and $E(k)$ correspondingly $[8,17]$ :

$$
J=\sqrt{F \xi_{2}}\left[\left(\xi_{1}+\xi_{2}\right) E(k)-2 \xi_{1} K(k)\right] / 3, \quad k=\sqrt{1-\xi_{1} / \xi_{2}} .
$$

If in the formula (13) the expression (14) of $J$ is expanded in powers of $F$ then one gets

$$
w=\frac{Z^{2}}{n^{3} n_{1} !\left(n_{1}+m\right) !}\left(\frac{4 Z^{3}}{n^{3} F}\right)^{2 n_{1}+m+1} \exp \left[-\frac{2 Z^{3}}{3 n^{3} F}-3\left(n_{1}-n_{2}\right)\right],
$$

which recovers the Slavyanov's result [14] at $Z=1$. If the next term in the expansions (6), (7) is taken into account allows to find the formula (44) obtained in [13] got within the etalon equation method is recovered.

The comparison of the figures obtained for the ionization rate of the ground state of the hydrogen atom from (13), (15), and the formula (44) of [4], respectively, with the numerical result of [13] shows that the increase of the field strength $F$ from 0.020 to 0.075 a.u. leads to an increase of the relative error from 0.7 to $14 \%$ for (13), from 11 to $74 \%$ for (15), and from 1.3 to $17 \%$ for (44) [4], i.e. formula (13) is more accurate than (15) and even (44) [4] which contains the correction of the order of $F$.

For excited states, the differences between the relative errors of (13) and each of (15) and (44) [4] are even more significant than for the ground state, except for the state $n_{1}=n_{2}=0, m=1$, for which the last formula gives accidentally very good result. Obviously, such high accuracy of the formula (13) is connected with use of the exact expression of the barrier integral which takes partially into account higher-order corrections in $F$, which are neglected in (15) and (44) [4].

To finding the tunneling ionization rates of singly charged negative ions (like $\mathrm{H}^{-}, \mathrm{J}^{-}$etc.), in (15) it is necessary to put $Z=0$. If the particle is in a weakly bound state in a central field with small radius of action $r_{0}$, then beyond this radius the asymptotic behaviour of the unperturbed $(F=0)$ radial wavefunction is of the form [1]

$$
R_{l m}^{(\mathrm{as})}=b r^{-1} e^{-\gamma r},
$$


where $b$ is determined by imposing the normalization condition. When $r_{0} \ll 1$ the behaviour of the wavefunction within the potential well $0 \leqslant r \leqslant r_{0}$ is not essential because the particle stands basically beyond the well. This gives $b \approx \sqrt{2 \gamma}$ and the ionization rate

$$
w=\frac{b^{2}(2 l+1)}{m ! \gamma^{m}} \frac{(l+m) !}{(l-m) !}\left(\frac{F}{4 \gamma^{2}}\right)^{m+1} e^{-2 \gamma^{3} / 3 F} .
$$

For $s$-states the formula (17) coincides with the known result of Demkov and Drukarev $[1,18]$.

\section{Quasiclassical solutions of the relativistic problem of the atom in a constant uniform electric field}

The difficulty in deriving a solution to the relativistic problem of the atom in a the constant uniform electric field related to the fact that the Dirac equation with the potential (1) does not permit complete separation of variables in any orthogonal system of coordinates. To solve this problem the combination of the quasiclassical approximation with boundary layer method [11] is used. Hereinafter we shall call such an approach the "method of quasiclassical localized states" (MQLS).

The main idea of this method is as follows. We construct the solution of the Dirac equation in the under-the-barrier range, where, in contradistinction to the case of the classically allowed range, the wavefunction is often localized in the vicinity of the most probable tunneling direction. This substantially simplifies the whole problem since the coordinate $\rho$ perpendicular to the $z$-axis plays the role of the small parameter with respect to which power series expansions can be used for all the quantities entering the equations obtained after the expansion of the solution in powers of $\hbar$. The coefficients of these series satisfy equations that can be solved exactly except for the Riccati equation which is solved approximately [11].

The effective potential

$$
U_{\text {eff }}(z, \varepsilon)=\varepsilon V_{0}-V_{0}^{2} / 2 c^{2}, \quad V_{0}(z)=-Z / z-F z, \quad \varepsilon=E_{\text {rel }} / c^{2}
$$

corresponding to this problem has a form which is similar to that of the potential with barrier in the non-relativistic case (Sec. 2). Here $E_{\text {rel }}$ is the relativistic energy of the electron, $c$ is the velocity of light.

If $F \ll \lambda^{4} / 4 Z\left(\lambda=c \sqrt{1-\varepsilon^{2}}\right)$ then the range $z_{1} \ll z \ll z_{m}\left(z_{1}\right.$ is the left tuning point, and $z_{m}$ is the maximum point of barrier) exists where one can match the WKB solution with the asymptotic (at large $z$ and small $\rho$ ) behaviour of the relativistic wavefunction $\Psi_{n, j, l, m_{j}}(l=j \pm 1 / 2)$ of the H-like atom with perturbed energy [6]

$$
E_{\mathrm{rel}}=E_{0}+\operatorname{sgn} \varkappa \frac{3}{4} \sqrt{N^{2}-\varkappa^{2}} \frac{\left(n_{r}+\gamma_{\mathrm{rel}}\right) m_{j} F}{j(j+1) Z} .
$$

Here $E_{0}=c^{2} / \sqrt{1+\left[Z \alpha /\left(n_{r}+\gamma_{\mathrm{rel}}\right)\right]^{2}}$ is the energy of the non-perturbed relativistic H-like atom, $N=\sqrt{n^{2}-2 n_{r}\left(|\varkappa|-\gamma_{\text {rel }}\right)}, \varkappa=(-1)^{j-l+1 / 2}(j+1 / 2), n_{r}=n-j-1 / 2, \gamma_{\text {rel }}=\sqrt{\varkappa^{2}-(Z / c)^{2}}$.

Having the normalized wavefunction in the under-the-barrier region and using the Zwaan rule [1] one can continue it into the classically allowed region $z>z_{2}\left(z_{2}\right.$ is the right tuning point for the effective potential (18)). Calculating the total probability flux through the plane which is perpendicular to the $z$-axis and located in the domain $z>z_{2}$, we obtain:

$$
w=\frac{2 \lambda_{0} A^{2}}{\left(1+\varepsilon_{0}\right)\left(\left|m_{j}\right|-1 / 2\right) !} \frac{\left(j+\left|m_{j}\right|\right) !}{\left(j-\left|m_{j}\right|\right) !}\left(\frac{Z}{2 \lambda_{0}^{2} e}\right)^{\frac{2 \varepsilon_{0} Z}{\lambda_{0}}}\left(4 \lambda_{0}^{2} \int_{z_{1}}^{z_{2}} \frac{d z}{q_{0}(z)}\right)^{-\left|m_{j}\right|-1 / 2} e^{-2 \int_{z_{1}}^{z_{2}} q_{0}(z) d z-2 Z \alpha \arccos \varepsilon_{0}},
$$

where $q_{0}=\sqrt{2\left(U_{\text {eff }}-E_{\text {eff }}\right)}, E_{\text {eff }}=-\lambda^{2} / 2$ is the effective energy, $\varepsilon_{0}=E_{0} / c^{2}, \lambda_{0}=c \sqrt{1-\varepsilon_{0}^{2}}$, 


$$
A=\sqrt{1+\varepsilon_{0}} \lambda_{0}\left(2 \lambda_{0}\right)^{\varepsilon_{0} Z / \lambda_{0}}\left(\frac{Z / \lambda_{0}-k}{2 Z n_{r} ! \Gamma\left(2 \gamma_{\mathrm{rel}}+n_{r}+1\right)}\right)^{1 / 2},
$$

where $k=\varkappa$ for the ground state and state with $l=j-1 / 2 ; k=0$ for all other states.

The integrals in (20) can be approximately calculated similar to the non-relativistic case. Omitting details of these calculations, we write the final result for the ionization rate:

$$
w=\frac{2 \lambda_{0} A^{2}}{\left(1+\varepsilon_{0}\right)\left(\left|m_{j}\right|-1 / 2\right) !} \frac{\left(j+\left|m_{j}\right|\right) !}{\left(j-\left|m_{j}\right|\right) !} \frac{e^{2 Z \alpha \arccos \varepsilon_{0}}}{\left(2 c \arccos \varepsilon_{0}\right)^{\left|m_{j}\right|+1 / 2}}\left(\frac{2 \lambda_{0}^{2}}{F}\right)^{\frac{2 \varepsilon_{0} Z}{\lambda_{0}}-\left|m_{j}\right|-1 / 2} \exp \left\{-\frac{c^{3} \Phi(\varepsilon)}{F}\right\},
$$

where $\Phi(\varepsilon)=\arccos \varepsilon-\varepsilon \sqrt{1-\varepsilon^{2}}$. The formula (22) differs from the result of [9] by the asymptotic coefficient $A$ which was obtained in [9] using the asymptotic behaviour of the Coulomb wavefunction within the Klein-Gordon equation. For the ground state the expression (22) coincides with the result of [10] obtained by means of the relativistic version of the ADK theory.

\section{Conclusion}

A recurrent scheme for finding the quasiclassical solutions of the one-dimensional equation obtained after separation of variables of the Schrödinger equation in parabolic coordinates was described. The method of quasiclassical localized states was developed for the Dirac equation with arbitrary axially symmetric potential which does not allow complete separation of variables. These approaches are based on physically clear ideas. They allowed the derivation of wavefunctions and of general analytical expressions for the leading term of the asymptotic behaviour of the ionization rate of the $\mathrm{H}$-like atom in a uniform electrostatic field in the non-relativistic and relativistic cases when the intensity of the electric field $F$ is much smaller than the intensity of the intra-atomic field.

\section{References}

[1] L. D. Landau, E. M. Lifshitz, Quantum Mechanics: Nonrelativistic Theory (Oxford Univ. Press, Oxford, 1975)

[2] H. A. Bethe and E. E. Salpeter, Quantum Mechanics of One- and Two-Electron Atoms (Springer-Verlag, Berlin, 1957)

[3] B. M. Smirnov, Physics of Atoms and Ions (Springer, New York, 2003)

[4] R. J. Damburg, V. V. Kolosov, in: Rydberg States of Atoms and Molecules, edited by R. F. Stebbings and F. B. Dunning (Cambridge University Press, Cambridge, 1983)

[5] R. C. Kulkarni, N. V. Swamy, and E. Chaffin, Phys. Rev. A 7, 27-33 (1973)

[6] S. A. Zapryagaev, Optika i spektroskopiya 44, 892-899 (1978)

[7] S. A. Zapryagaev and N. L. Manakov, Yad. Fiz. 23, 917-925 (1976)

[8] V. Yu. Lazur, O. K. Reity, and V. V. Rubish, Int. J. Mod. Phys. A 25, 3235-3259 (2010)

[9] V. D. Mur, B. M. Karnakov, and V. S. Popov, JETP 87, 433-444 (1998)

[10] N. Milosevic, V.P. Krainov, and T. Brabec, J. Phys. B 35, 3515-3530 (2002)

[11] O. K. Reity, V. Yu. Lazur, and A. V. Katernoha, J. Phys. B 35, 1-17 (2002)

[12] H. J. Silverstone, Phys. Rev. A 18, 1853-1864 (1978)

[13] R. J. Damburg and V. V. Kolosov, J. Phys. B 11, 1921-1930 (1978)

[14] S. Yu. Slavyanov, Problems of Mathematical Physics [in Russian] 4, 125 (1970)

[15] T. Yamabe, A. Tachibana, and H. J. Silverstone, Phys. Rev. A 16, 877-890 (1977)

[16] B. M. Smirnov and M. I. Chibisov, Sov. Phys. JETP 22, 585-592 (1966)

[17] V. Yu. Lazur, O. K. Reity, and V. V. Rubish, Theor. Math. Phys. 155, 825-847 (2008)

[18] N. N. Demkov and G. F. Drukarev, Soviet Phys. JETP 20, 614-618 (1965) 